\title{
Enantioseparation of D- and L- isomers of Chiral Drugs for Improving their Bioavailability: Some Techniques Including Micellization with Gemini Surfactants
}

\author{
Nirmal Singh ${ }^{1 *}$, Lalit Sharma ${ }^{2}$ \\ ${ }^{1}$ Department of Chemistry, RSD College, Ferozepur, INDIA. \\ ${ }^{2}$ Department of Applied Sci. and Humanities, Shaheed Bhagat Singh State Technical Campus, Ferozepur, INDIA.
}

\begin{abstract}
The enantiopure drugs are essential for disease treatment as the human body is amazingly chiral selective. Nearly $50 \%$ of drugs are chiral but the pharmacological activity resides with only one isomer, termed as eutomer, whereas the other isomer which is inactive or less potent metabolizes by a different way in the body. This toxic isomer in a racemic drug causes side-effects, genetic disorder or may cause even death if taken in high dosage. Therefore, role of stereochemistry in drug action getting more and more attention of medical practice and one should be good knowledge to make decisions regarding while using single enantiomer of any drug. Most of the drugs used in psychiatric practice are currently available in mixture of enantiomers. For some therapeutics, one particular isomer of chiral drug give better pharmacological results with respect to the target organ, bioavailability in body plasma, low toxicity etc., as compared to racemic mixture of that drug. This article explains the chirality of drugs, the stereochemistry of isomers of chiral drugs, emphasizes the difference in the potential biological and pharmacologic differences between the two enantiomers of a drug, and highlights the novel technique to increase the in-vivo solubility of particularly one isomer of drug, so that the bioavailability of eutomer can be enhanced. This can be done by used of surfactants, which encapsulate the enantiomers of drugs at different extend by micellization.
\end{abstract}

Key words: Enantioseparation, Micellization, Chiral drugs, in-vivo bioavailability, Pharmacological activity.

\section{INTRODUCTION}

The Chirality term was originated and discovered by Louis Pasteur for sodium ammonium tartrate in 1848, and further it was found that most of the biomolecules i.e. carbohydrates, amino acids, nucleosides are chiral in nature. Chirality is the property of chiral compounds, which is due to the presence of chiral carbon in the organic compound i.e. a carbon atom which is attached to four different. Secondly, the compound should be asymmetric in nature so that the mirror image of the compound should not be superimposable with its mirror image. The compounds which are superimposing its mirror image are referred as achiral, and such compounds do not show optical activity. Chirality also found everywhere in our body, including the main building blocks of body i.e. amino acids, proteins and also in carbohydrates, nucleic acid (nucleobases), lipid etc. Chirality is explained as - isomer or dextro-isomer (the isomer which rotate the plane polarized light toward right side) and -isomer or laevo-isomer (which rotate the plane polarized light towared left side). These - and - isomers are termed as enantiomers and they are not super imposable to each other. The equimolar mixture of -
Submission Date: 11-11-2017; Revision Date: 24-01-2018; Accepted Date: 02-03-2018

DOI: 10.5530/ijper.52.3.39 Correspondence: Nirmal Singh, Department of Chemistry, RSD College, Ferozepur City-152002 (Pb.), INDIA. Phone: 9501526900 E-mail: nirmalsinghsekhon2012@gmail.com

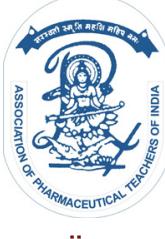

www.ijper.org 
isomer and - isomer is known as racemic mixture. But, now days, d- and l- system of designation of chiral compound is considered as old system, so new absolute configuration designated as $\mathrm{R} / \mathrm{S}$ - system of nomenclature for optical rotation is used. The chiral carbon of optical active compound is designated as R- (in Latin rectus right side) or S- (in Latin sinister - left side), in order to describe the stereochemistry of that chiral compound. The R- and S- designation are given by certain rules, based on the priority order of atoms attached to chiral carbon, that priority is given according to their atomic number or atomic masses (in case atomic number is identical). After assigning priority order to all the four atoms or group of atoms, rotate eyes from highest priority group to lowest, in doing so, if eyes rotate towards right, it is considered as R-and if eyes rotate toward left, it is considered as S- isomer, provided the lowest priority group should be away from the observer. If any organic compound or chiral drug contains more than one chiral centre, then the stereochemistry of all the chiral centers should be considered before assigning the final optical activity of that drug or compound. Optical rotation is denoted as $(+)$ for dextrorotatory, (-) for laevorotatory and for racemic mixture. So, enantiomers come in pairs. Both the isomer may have same physical and chemical properties, but differ in rotation of plane polarized light: either to left direction or to right direction. So, these two isomers interact differently in our body. As receptors, enzymes in the body are chiral too, so they behave different way toward chiral drug / compound. Single enantiomers either - or -are also called single isomer or stereoisomer. If that single stereoisomer is active against a particular disease that isomer is called eutomer.

\section{Importance of Chirality in Drug Molecules}

Only one enantiomer is responsible for all pharmaceutical activities. The therapeutic inactive isomer is considered as a foreign substance which is responsible for undesirable pharmacological effects. But if the active enantiomer exhibits a poor therapeutic value or show toxic behavior, the situation may become even more acute viz. $\mathrm{R}$ - and $\mathrm{S}$ - enantiomers of thalidomide (Figure 1) (the $\mathrm{R}$ and $\mathrm{S}$ designation is absolute configurations, by Cahn, Ingold and Prelog, is a way of naming enantiomers by their structures) is a therapeutic-specific pair. The R-enantiomer of this drug is therapeutically active and cause sedation, calming and soothing effect that relieves anxiety and promotes sleep. But, the S-enantiomer of thalidomide may cause teratogen formation, and this was reported to causes serious birth abnormalities in about 2000 children.

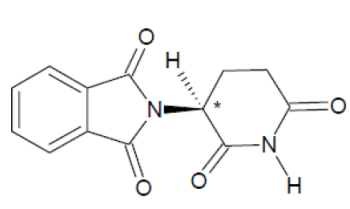

$(-)(S)$-thalidomide

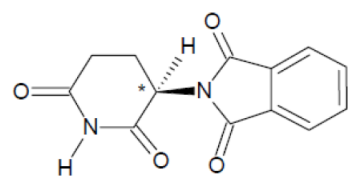

$(+)(R)$-thalidomide
Figure 1: The enantiomers of thalidomide.

There are many other drugs in which one particular enantiomer is active or more potent towards required therapeutic action. Ethambutol: Whereas the $(\mathrm{S}, \mathrm{S})-(+)$-enantiomer is used to treat tuberculosis, the $(\mathrm{R}, \mathrm{R})-(-)$ Ethambutol causes blindness. Naproxen: (S)$(+)$-naproxen act as analgesic and used to treat arthritis pain, but (R)-(-)-naproxen causes side effects such as liver poisoning. The receptor in the body is also responsible for therapeutic effect such as Penicillin drug is stereo dependent, it act as per the behavior of receptor in the body. This antibiotic must entre by interacting with D-alanine chain of cell walls of bacteria in order to react with and inhibit the transpeptidase enzyme.

Propranolol: the L-isomer of propanolol is a powerful adrenoceptor antagonist, but D-isomer is inactive. However, both isomers are active when they are used as a anesthesia. Methorphan: The L-methorphan which is termed as levomethorphan, is a active opioid analgesic, but, the D-methorphan which is termed as dextromethorphan, is suppress cough actively. Carvedilol: (S)-(-)-isomer of carvefilol is 100 times more potent toward interaction with adrenoceptors as compared to its $(\mathrm{R})-(+)$-isomer. The S-enantiomer of ofloxacin was found to be reported as 8 - to 128 -fold more active against both gram-positive and gram-negative bacteria than the R-antipode. ${ }^{1}$

\section{Drugs used as Racemates}

Most of the pharmaceutical compounds are marketed as recemates, mixture of two enantiomers of chiral drugs, each with its own chemistry, receptor affinity and pharmacokinetic profile. Two such drugs are albuterol for asthma and omeprazole for gasteroesophageal reflux disease and peptic ulcer were reported. ${ }^{2}$ with improved efficacy, pharmacokinetic and reduced side effects and with almost no drug-drug interaction, by using single isomer instead of racemic mixture. But, sometimes, to get optimal activity, some of them need to be used as recemates i.e. labetalol and nebivolol. Many of the recemates need to be separated into single enantiomer or chirally pure components prefixed as $\mathrm{R}$ or $\mathrm{S}$ enantiomer. 


\section{Classification of drugs: bases on the activity of particular enantiomers}

All drugs produce side effects, as the extent of toxicity varies widely between different enantiomers. Many toxic reactions which cause side effects due to their therapeutic effect can be avoided by changing drug dose. Recent interest has focused on the role of the different properties of individual drug enantiomers in causing drug toxicity. The drug which has one chiral carbon, exist in the form of pair of isomers, known as enantiomers, both isomer may be active pharmacologically. However, if the main therapeutic benefit resides with one isomer, several possibilities exist for the other enantiomer - the second enantiomer may be inactive, show qualitatively different effect, an antagonistic effect or greater toxicity. ${ }^{3}$

I. Drugs in which one enantiomer is active while the other enantiomer is inactive are: S-atenolol is more potent than R-atenolol towards beta blocking property, Levocetrizine - which is antihistamine, R-enantiomer (levo) is more potent than S-isomer; levofloxacin-antibacterial activity resides in the S-enantiomer only. In some cases, the $\mathrm{R}$ enantiomer is more potent than $\mathrm{S}$ viz. R-Pantoprazole, R-Metoprolol.

II. Drugs in which one isomer is more potent than the other are: Methylphenidate: $(\mathrm{R}, \mathrm{R})$-isomer of methylphenidate-approximately is about ten times more potent than (S,S)-methylphenidate; Ondansetron: R-ondansetron - more potent than the S-enantiomer; Pantaprazole: S-pantoprazole - more potent than the R-enantiomer.

III. Drugs in which beneficial effects reside in one enantiomer, the other enantiomer having antagonistic activity are: Salbutamol: (S) - isomer of this drug is responsible for the bronchodialator activity, but it indirectly involved in antagonizing the benefits of (R)-salbutamol and may have proinflammatory effect; Lipoic acid: the beneficial effects reside with R-lipoic acid, while the corresponding S-form can oppose the action of its R-form.

IV. There are many drugs in which enantiomers have entirely different therapeutic effects. For example; $\mathrm{R}$-isomer of Fluxetine is used to cure depression while $\mathrm{S}$-isomer is effective for treating migraine; S- Propanolol act as -blocker and membrane stabilizer, whereas $\mathrm{R}$-isomer of propanolol has membrane stabilizing as well as spermicidal properties and can be used to treat hyperthyroidism; R-Sibutramine is effective in treating depression whereas S-Sibutramine is used for curing ejaculatory and erectile dysfunction. Some drugs are there in which only one enantiomer is responsible for beneficial effects whereas the other enantiomer has adverse activity are: S- Amlodipine is a calcium channel blocker (CCB) while R-isomer is inactive as CCB.

\section{Chiral Switching: Switches to active isomer from racemates}

Use of single enantiomer instead of racemic mixture of some particular chiral drug, is termed as chiral switching. It involves development of unichiral form of the racemic drug which is already in the market. This is one of the methods to get safe-alternative of drug which exist as racemates. Many of novel single-enantiomer of drugs was developed as such, but there are also important examples of new single-enantiomer drugs derived ${ }^{4}$ from 'chiral switches' of established racemates.

\section{Methods to get single safer enantiomers of chiral drugs}

(a) Single isomer chemical entity (NSCE): the drugs which are developed as a single enantiomers come under this category i.e. enalapril, ramipril, diltiazem, atorvastatin, simvastatin, pravastatin, clopidogrel, L-carnitine, levodopa, d-penicillamine, levetiracetam, and rivastigmine.

(b) Actual chiral switches: Switching from existing racemic drug to one of its isomers to get safer alternatives, termed as chiral switches, in other words, these are the drug which were initially marketed as racemic mixtures, but after that one isomer is isolated according to their useful therapeutic effects in our body i.e. escitalopram, esomeprazole, dexibuprofen, dexketoprofen, S-ketamine, levocetirizine, levofloxacin, $(\mathrm{R}, \mathrm{R})$-methylphenidate, levo-leucovorin, levo-bupivacaine, and eszopiclone. Switching from racemates to single isomer covers a wide range of drug categories, such as cetrizine which is antihistamines and ketamine which is anaesthetics. There are some examples of chiral switches done recently to produce more safe and more potent alternative to the existing racemates viz. S-isomers of atenolol, metoprolol, ketamine, amlodipine, zopiclone, omeprazole and pantoprazole, and R-isomer of ondansetron, levosalbutamol, levobupivacaine etc. Chiral switch found to replace many racemate drugs to their safer single-isomer drugs, which are more potent and causes low toxicity.

Emcure Pharmaceuticals Limited, Pune is reported to develop a number of single enantiomer (unichiral) drugs, e.g. S-isomers of amlodipine, atenolol, metoprolol, pantoprazole, and R-isomer of ondansetron.

\section{Single enantiomers drugs}

\section{The commonly used single-enantiomer drugs are} classified as:

Respiratory drugs which are used to prevent, relieve or treat the respiratory diseases viz. Fluticasone propionate 
(a synthetic trifluorinated glucocorticoid receptor agonist with antiallergic, antiinflammatory and antipruritic effects) and Salmeterol (used in case of severe asthma) Hematology includes the problems with red blood cells, white blood cells, platelets, bone marrow, lymph node etc. viz. Clopidogrel bisulfate commercially known as Plavix, it inhibits platelets aggregation and blood clotting. Gastrointestinal drugs used to treat gastrointestinal disorder in body i.e. Esomeprazole magnesium commercially known as Nexium, used to treat stomach and esophagus problems.

Antibiotic drugs also called antibacterial drugs, used for prevention and treatment of bacterial infection i.e. Amoxicillin, potassium clavulanate and Levofloxacin

Cardiovascular drug is used to treat medical condition related to heart and blood vessels i.e. Atorvastatin calcium (used to reduce bad cholesterol) Simvastatin (as lipid lowering medication), Pravastatin sodium and Valsartan

Central nervous system drugs are used to treat anxiey and as sleep aids i.e. Paroxetine hydrochloride, Sertraline hydrochloride and Armodafinil

Antihypertensive and Antianginal agent: Levamlodipine Pediatric anesthesia: Levobupivacaine

Antiallergic: Levocetrizine

Antihistiamine drug: Dexachlorpheniramine

Antidepressant drugs: Escitalopram, Levomilnacipran

Bronchodilator: Arformoterol

Analgesic: Dexibuprofen

Antidepressant: Esketamine

\section{Methods for separation of enantiomers of chiral drugs: Enantioseparation}

Various laboratory techniques are available for enantioseparation. ${ }^{5,6,7,8}$ Typically, for very broadly applicable laboratory techniques such as chromatography. ${ }^{9}$ and capillary electrophoresis ${ }^{10}$ high capital investments are required. ${ }^{11}$ But still, it is found that for small volumes of single enantiomers needed in early development stages, the high enantioseparation costs by chromatographic

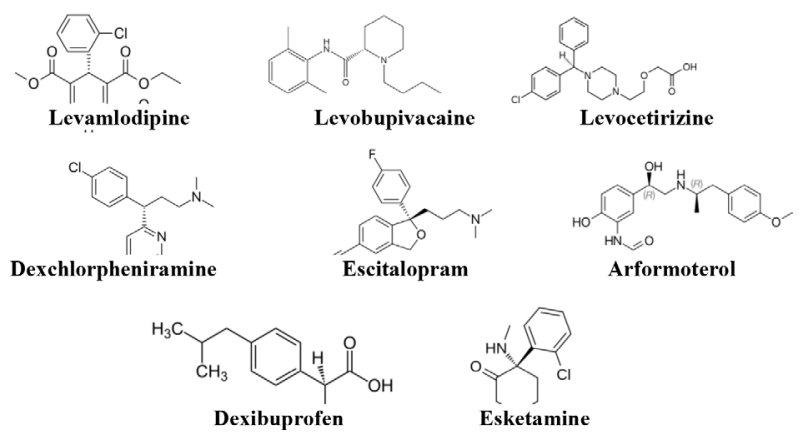

Scheme 1: Structure of some single enantiomer drugs methods were still less as compare to the total cost for drug development. ${ }^{12}$ Some impressive preparative chiral separations have been demonstrated using centrifugal partition chromatography ${ }^{13}$ and simulated moving bed chromatography. ${ }^{14,15}$ Another strategy for chiral separations is based on the use of membrane-based approaches. ${ }^{16-20}$ When applying immobilized selectors in (liquid) membranes, the amount of selector needed can be reduced greatly. Low transport rates through the membranes are the main limitations of this technology.

To achieve a high transport rate, liquid-liquid extraction, diffusion and convection transport mechanisms are used. ${ }^{21}$ Liquid-liquid extraction is a well known technology which is easy to operate to fractionate the racemate into its enantiomers. ${ }^{22}$ The liquid-liquid extraction can be used for wide range of material, as from small scale such as laboratory separation to bulk scale such as separation in chemical industry, so liquid -liquid extraction is one of the most popular technique used for enantioseparation and is known since 1959. ${ }^{23}$ The detail regarding first liquid-liquid enantiosepartion was found in late 1960 's. ${ }^{24,25}$

\section{Capillary Electrophoresis}

Capillary electrophoresis. ${ }^{26}$ is an analytical technique based on the ionic mobility under the influence of applied electric field. The electrophoretic mobility is dependent upon the charge of the ion, the viscosity, and the atom's radius. The separation of ions depends upon the magnitude of applied electric field, more the field strength, faster the separation of ions. Only ions respond toward electric field, so neutral entities are not affected. The mobility of ion also depends upon the charge carrying by that ion, more the charge more will be the mobility of that particular ion under the influence on applied electric field. Capillary electrophoresis is one the most predominately used technique for the separation of ions as it gives results faster with high accuracy.

The enantioseparation of three chiral drugs with the help of capillary electrophoresis was reported. ${ }^{27}$ In this method, an achiral ionic liquid, 1-butyl-3-methylimidazolium chlorine ([BMIM] $\mathrm{Cl}$ ), is used as an additive and $\beta$-cyclodextrin (beta-CD) was used as a chiral selector, the enantiomers of chlorpheniramine, the precursor of Chloramphenicol and of Loxacin were separated by capillary zone electrophoresis.

Capillary zone electrophoresis was used for enantiomer separation of set of 59 chiral drugs. ${ }^{28}$ Six enantiomeric pair of chiral drugs was separated by using $\alpha$-cyclodextrin as chiral solvating agent. Intially, the cildinium bromide, oxomemazine and tetryzoline were separated, and then 
ketamine, orphenadrine, tropicamide were separated by futher optimization of the process.

The capillary electrophoresis method of separation of drug enantiomers using proteins as the chiral selectors was reported ${ }^{29}$ The proteins used in this electrophoresis method was albumins such as bovine serum albumin, human serum albumin and serum albumins from other species, glycoproteins such as $\alpha_{1}$-acid glycoprotein, crude ovomucoid, ovoglycoprotein, avidin and riboflavin binding protein, enzymes such as fungal cellulase, cellobiohydrolase I, pepsin and lysozyme and other proteins such as casein, human serum transferrin and ovotransferrin.

\section{Chromatography: High Performance Liquid Chromatography (HPLC)}

In the HPLC, racemate solutes distribute between the stationary phase and the mobile phase through a combination of sorption and desorption processes. The process slows down the advancement of the enantiomers as they pass through the separation column. The enantiomers are slowed down to varying degrees depending on their affinity for the stationary phases. So, this way separation of enantiomers can be done. From many years, the considerable interest had been developed for synthesis and separation of enantiomers of organic compounds especially HPLC method was used for the separation of enantiomers of drugs, because of their importance in the biochemistry and pharmaceutical industry. ${ }^{30}$ From last 40 years many novel chiral stationary phases for gas and liquid chromatography was developed, which were found to be very effective in separation of enantiomers and other various chiral compounds. But still, separation of enantiomer of drugs which have more than two chiral centers was challenging. The separation of enantiomers of reboxetine by HPLC was reported by using cellulose Tris (3,5-dimethyl phenyl) carbamate on silica gel. Various concentration mixture of $\mathrm{n}$-hexane and propan-2-ol were studied as a mobile phase, the optimum result was reported by using a specific concentration of $\mathrm{n}$-hexanol and propan-2-ol of $80 \%$ by volume. ${ }^{31}$ Enantioseparation of a compound with three chiral centres viz. nadolol, was obtained by HPLC on a column packed with heptakis (6-azido6-deoxy-2, 3-di-O-phenyl carbamolyted)-cyclodextrin bonded chiral stationary phase. After the separation of three enantiomers of nadolol, the (RSR)-nadolol was found to be the most active form of drug. ${ }^{32}$

\section{Crystallization}

Crystallization is one of the physical separation technique used for enantioseparation. Besides its application in resolution of racemates, it can also be used in the enrichment of partially pure isomers obtained from asymmetric synthesis, biotransformation kinetic resolution and HPLC. There are three main categories of crystallization for enatiometirc resolution:

Preferential crystallization or separation of enantiomers by stereospecific growth of each enantiomer in different crystallizer. This process requires no resolving agent. To describe the enantioseparation by preferential crystallization in coupled crystallizer a mathematical model was reported. ${ }^{33}$

Stereoisomeric crystallization- the reagent which is used as resolving reagent form different salts with the pair of enantiomers. These salt formed can be resolved according to their different phase behavior. A novel method, stripping crystallization (SC), has been reported. ${ }^{34}$ to separate the isomers of ibuprofen.

Catalytic kinetic resolution-in this method of resolution, the reagent used combine with different rate with each enantiomer. A novel co-crystallization in solution method was reported ${ }^{35}$ for resolving racemic mixture.

\section{Micellization by Surfactants: Enhancing in vivo bioavailability}

As surfactants have dualistic property, the head is polar (hydrophilic) so soluble in aqueous solution, while the tail is non-polar (hydrophobic), so insoluble in aqueous medium but soluble in non-aqueous Solvents. When surfactants are added to water, at some particular concentration they aggregate to form micelle. The process is called micellization, and the concentration at which micelles are formed is termed as Critical Micellization Concentration (CMC). Drug is encapsulated inside the micelle (Figure 2) as, drug is hydrophobic.

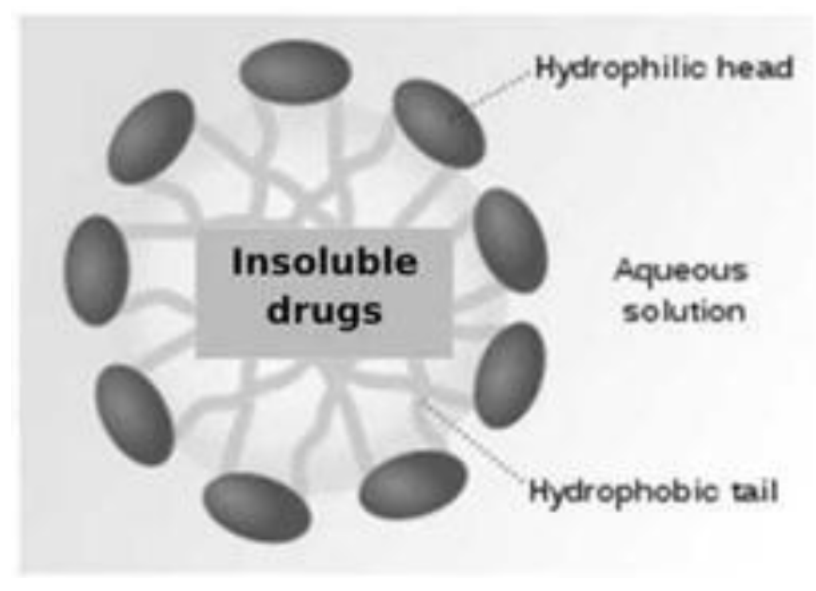

Figure 2: Drug encapsulating in micelle. 


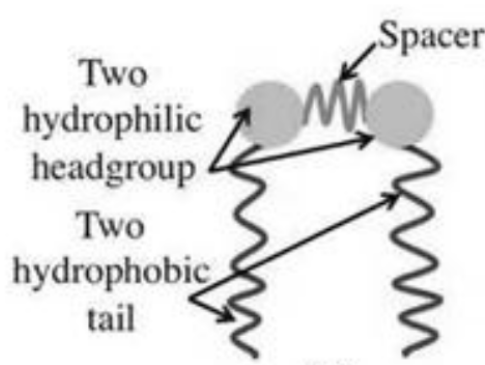

(a)

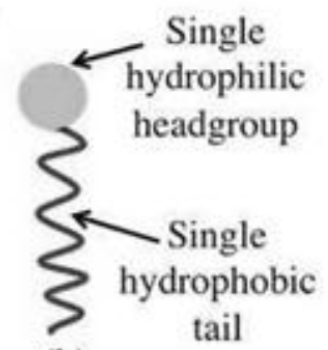

(b)
Figure 3: (a) Gemini surfactant and (b) conventional surfactant.

\section{Surfactants and Gemini surfactants Introduction}

Gemini surfactants are novel class of amphiphiles molecules, first reported in $197 .{ }^{36}$ Gemini amphiphile (Figure 3a) molecules consist of two conventional surfactants connected by a flexible. ${ }^{37}$ or rigid ${ }^{38}$ spacer, which can be hydrophilic or hydrophobic. ${ }^{39}$ Depending on nature of head group, geminis may be anionic, cationic, non-ionic or zwitterionic. They typically show better aggregation behavior as compared to the corresponding monovalent (single chain, single head group) or conventional surfactant (Figure 3b). Surface activity can be increased 1000-fold.

\section{Encapsulation by micellization using non-ionic Surfactants/non-ionic Gemini Surfactants}

These surfactants and gemini surfactants were explored to encapsulated poorly water soluble drugs, in order to enhance their water solubility and thus their bioavailability in the body plasma. The gemini surfactant has low critical micellization concentration than conventional surfactants, so they have many fold more tendency to encapsulate the drug moieties. In one of our study, solubility of poorly water soluble drugs viz. norfloxacin, glibenclamide and clofibrate enhanced many folds by micellization with nonionic gemini surfactants. ${ }^{40}$ It was found that nonionic gemini surfactants encapsulate the hydrophobic drugs in the order Clofibrate $>$ Norfloxacin $>$ Glibenclamide. It was also found that more hydrophobic surfactants with longer alkyl tails give better micellar ratio for the encapsulation of drugs molecule, which is better recognized in order of their smaller size, i.e., the smaller is the size of drug molecule, the better is its encapsulation. The solubility of poorly water soluble drugs i.e. Glibenclamide, Clofibrate and Norfloxacin was enhanced many fold by micellization in the presence of carbohydrate derived non-ionic surfactants. The aqueous solubility of Glibenclamide was enhanced 10.8 times, that of Clofibrate was enhanced about
16.8 times $^{41}$ and that of Norfloxacin ${ }^{42}$ was enhanced by 16.4 times, this potentially increases their bioavailability.

\section{CONCLUSION}

Enantioseparation of chiral drugs by micellization is a novel approach to enhance the bioavailability of drug in the blood stream. Use of single isomer for the treatment of a particular disease is much safer / effective than to use racemic mixture. This reduces the side effect as well as enhances the therapeutic effect at the target cell in the body plasma. The novel synthesized carbohydrate derived surfactants or non-ionic gemini surfactants were explored for recognition of enantiomers of chiral drugs, as these surfactants encapsulate D- and L- enantiomers at different extent. The above discussed surfactants were used to encapsulate the poorly water soluble durgs i.e. Norfloxacin, Glibenclamide and Clofibrate, and enhanced the solubility of these drugs many folds. The increased solubility of these drugs in water, potentially improve their bioavailability, which is required to get desirable therapeutic effect of any particular drug in the body.

\section{ACKNOWLEDGEMENT}

We are grateful for financial support to Department of Science and Technology, Government of India, New Delhi (grant SERC/OC-17/2006). Spectroscopic analysis by SAIF, Panjab University, Chandigarh is highly appreciated

\section{CONFLCIT OF INTEREST}

The authors declare no conflict of interest

\section{ABBREVIATIONS}

CCB: Calcium Channel Blockers, NSCE: Single Isomer Chemical Entity, [BMIM]Cl : 1-butyl-3-methyLimidazolium Chloride, Beta-CD: Beta-Cyclodextrin, HPLC: High Performance Liquid Chromatography, SC: Stripping Crystallization, CMC: Critical Micellization Concentration.

\section{REFERENCES}

1. Atarashi S, Yokohama S, Yamazaki K, Sakano K, Imamura M, Hayakawa I. Synthesis and antibacterial activities of optically active ofloxacin and its fluoromethyl derivative. Chem Pharm Bull. 1987;35(5):1896-902.

2. Flockhart DA, Nelson HS. Single isomer versus race mate: is there a difference? Clinical comparisons in allergy and gastroenterology. CNS Specter. 2002;7(S1):23-7.

3. Scott AK. Stereoisomers and drug toxicity. The value of single stereoisomer therapy. Ther Adv Drug Safe. 1993;8(2):149-59. 
4. Agranat I, Caner H, Caldwell J. Putting chirality to work: the strategy of chiral switches. Nat Rev Drug Discov. 2002;1(10):753-68.

5. Subramanian G. Chiral Separation Techniques a Practical Approach WileyVCH: Weinheim. 2008

6. Toda F. Enantiomer Separation Fundamentals and Practical Methods: Kluwer Academic Publishers Dordrecht; 2004.

7. Guebitz G, Schmid MG. Chiral Separations: Humana Press Totowa (NJ). 2004.

8. Busch KW, Busch MA. Chiral Analysis by regression modeling of spectral data in chiral analysis. Elsevier: Amsterdam. 2006;363-95.

9. Davankov VA. Chiral selectors with chelating properties in liquid chromatography: Fundamental reflections and selective review of recent developments. J Chromatogr A. 1994;666(1-2):55-76.

10. Jira T, Bunke A, Schmid MG, Gubitz G. Chiral resolution of diols by capillary electrophoresis using borate-cyclodextrin complexation. J Chromatogr A. 1997;761(1-2):269-75.

11. Steensma M, Kuipers NJM, De Haan AB, Kwant G. Identification of Enantioselective Extractants for Chiral Separation of Amines and Aminoalcohols. Chirality. 2006;18(5):314-28.

12. Cox GB. Introduction to Preparative Chromatography in Preparative Enantioselective Chromatography (ed G. B. Cox). Blackwell Publishing Ltd: Oxford UK. 2005:19-47.

13. Gavioli E, Maier NM, Minguillon C, Lindner W. Preparative Enantiomer Separation of Dichlorprop with a Cinchona-Derived Chiral Selector Employing Centrifugal Partition Chromatography and High-Performance Liquid Chromatography a Comparative Study. Anal Chem. 2004;76(19):5837-48.

14. Francotte E, Leutert T, Vecchia LL, Ossola F, Richert P, Schmidt A. Preparative resolution of the enantiomers of Tert-leucine derivatives by simulated moving bed chromatography. Chirality. 2002;14(4):313-7.

15. Zenoni G, Quattrini F, Mazzotti M, Fuganti C, Morbidelli M. Scale-up of analytical chromatography to the simulated moving bed separation of the enantiomers of the flavour norterpenoids $\alpha$-ionone and $\alpha$-damascone. Flavour and Fragrance Journal. 2002;17(3):195-02.

16. Afonso CAM, Crespo JG. Angew Chem. Int Ed. 2004: 5293-5.

17. Keurentjes JTF, Nabuurs, LJWM, Vegter EA. Liquid membrane technology for the separation of racemic mixtures. J Membrane Sci. 1996;113(2):351-60.

18. Maximini A, Chmiel H, Holdik H, Maier NW. Development of a supported liquid membrane process for separating enantiomers of $\mathrm{N}$-protected amino acid derivatives. J Membrane Sci. 2006; 276(1-2):221-31.

19. Xie R, Chu LY, Deng JG. Membranes and membrane processes for chiral resolution. Chem Soc Rev. 2008;37(6):1243-63.

20. Maier N, Franco P, and Lindner W. Separation of enantiomers: needs, challenges, perspectives. J Chromatogr A. 2001;906(1-2):3-33.

21. Viegas RMC, Afonso CAM, Crespo JG, Coelhoso IM. Modelling of the enantio-selective extraction of propranolol in a biphasic system. Sep Purif Technol. 2007;53(3):224-34.

22. Dashkevic LB. Tr Leningr Khim-Farm Inst; 1959. p. 29.

23. Bauer K, Falk H, Schlogl K. Racematspaltung von chiralen Ferrocenderivaten durch Gegenstromverteilung, Monatshefte Fur Chemie. 1968;99(6):2186-94.

24. Romanko SJ, Wells KH, Rothbart HL, Rrieman W. Resolution of racemic substances by liquid ion-exchange. Talanta. 1969;16(5):581-90.
25. Schweitzer GK, Supernaw IR, Bowman NS. The solvent extraction resolution of some optically-active neutral chelates. J Inorg Nucl Chem. 1968;30(7):1885-7.

26. Sam Li. Capillary Electrophoresis: Principles, Practice, and Applications. J of Chromatography Library Elsevier Science Publishers. The Netherlands. 52. 1992.

27. Xia C, Chen Z, Xia Z. Separation of enantiomers of three chiral drugs by capillary electrophoresis has been reported. Sep Purif Technol. 2008;26(6):677-81.

28. Bingcheng Lin, Yibing Ji, Yuying C, Epperlein U, Koppenhoefer B. Separation of drug enantiomers by capillary electrophoresis: $\alpha$-cyclodextrin as chiral solvating agent. Chromatographia. 1996;42(1-2):106-10.

29. Haginaka J. Enantiomer separation of drugs by capillary electrophoresis using proteins as chiral selectors. J Chromatogr A. 2000;75(1-2):235-54.

30. Chamsaz M, Asadpour S, Yazdi AS, Ghasemi J. High-performance liquid chromatographic enantioseparation of drugs containing multiple chiral centers on chiral stationary phases. J Iran Chem Res. 2009;2:1-21.

31. Cannazza G, Braghiroli D, Carrozzo MM, Parenti C, Sabbioni C, Mandrioli $\mathrm{R}$, et al. Enantioseparation of the antidepressant reboxetine. J Pharm Biomed Anal. 2008;48:991-96.

32. Wang $\mathrm{X}$, Ching $\mathrm{CB}$. Liquid chromatographic retention behavior and enantiomeric separation of three chiral center $\beta$-blocker drug (nadolol) using heptakis (6-azido-6-deoxy-2,3-di-Ophenylcarbamolyted) $\quad \beta$-cyclodextrin bonded chiral stationary phase. Chirality. 2002;14(10):798-805.

33. Chaaban JH, Johansen KD, Skovby T, Kiil S. Separation of Enantiomers by Preferential Crystallization: Mathematical Modeling of a Coupled Crystallizer Configuration. Org Process Res Dev. 2014;18(5):601-12.

34. Shiau LD, Liu KF, Hsu YC. Chiral purification of S-ibuprofen from ibuprofen enantiomers by stripping crystallization. Chem Engg Res Design. 2017;117:301-8.

35. Springuel G, Leyssens T. Innovative Chiral Resolution Using Enantiospecific Co-Crystallization in Solution. Cryst Growth Des. 2012;12(7):3374-8.

36. Bunton CA, Robinson LB, Schaak J, Stam MF. Catalysis of nucleophilic substitutions by micelles of dicationic detergents. J Org Chem. 1971;36(16):2346-50.

37. Zana R, Talmon Y. Dependence of aggregate morphology on structure of dimeric surfactants. Nature. 1993;362(6417):228-30.

38. Menger FM, Littau CA. Gemini surfactants: a new class of self-assembling molecules. J Am Chem Soc. 1993;115(22):10083-90.

39. Zana R. Specialist surfactants in: Robb, I.D. (ed.) 81.1996. Champman Hall Ltd., London

40. Singh N, Sharma L. Synthesis and use of novel carbohydrate-derived nonionic surfactants for enhancing the aqueous solubility of norfloxacin by micellization. Trends in Carbohydrate Research. 2016;8:33-7.

41. Singh N, Sharma L. Enhancement in solubility of Glibenclamide and Clofibrate drugs using carbohydrate based non-ionic surfactants by micellization. Indo Am J Pharm Sci. 2017;4(2):186-92.

42. Singh N, Sharma L. Encapsulation of Poorly Water Soluble Drugs with Novel Non-Ionic Gemini Surfactants by Micellization. Annual Int'l Conference on Chemical Processes, Ecology and Environmental Engineering (ICCPEE'16) April 28-29, 2016 Pattaya (Thailand):6-9.

\section{SUMMARY}

Recent drug discovery has led to an increasing number of new drugs with low water solubility and hence poor bioavailability, especially via oral administration. The number of such drug candidates has increased enormously and almost $60 \%$ of the new drug candidates have shown poor aqueous solubility in the recent years.

Enhancement in their aqueous solubility may improve their bioavailability and thus, reduces side effect, and the dose of the drug can be reduced. Gemini surfactants are new category of surfactants which comprising two conventional surfactants connected via spacer. They have very low critical micellisation concentration (cmcs), so they can encapsulate drugs more effectively. Further, these gemini surfactants can be used for recognition of D- and L- enantiomers of chiral drugs. 


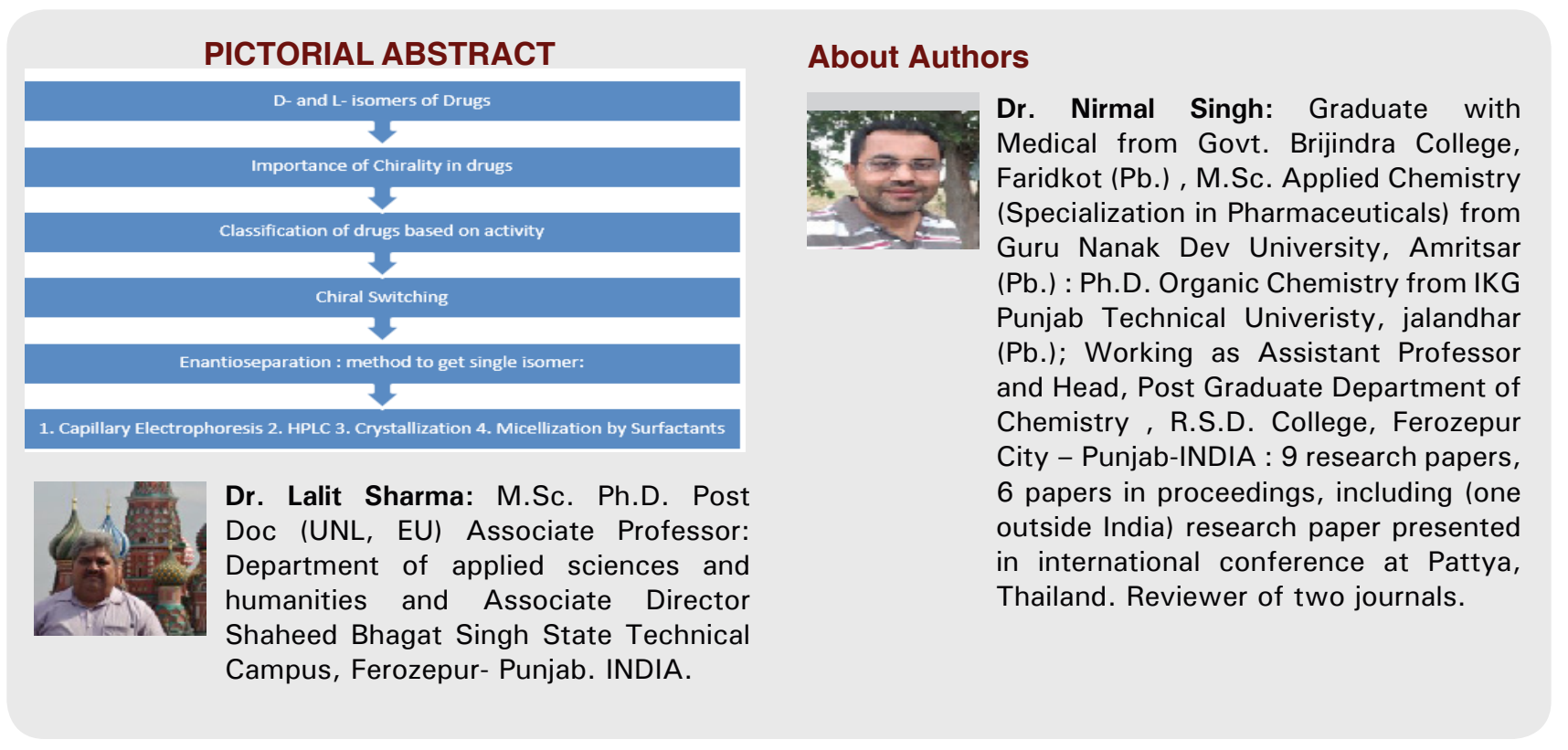

Cite this article: Singh N, Sharma L. Enantioseparation of D- and L- isomers of Chiral Drugs for Improving their Bioavailability: Some Techniques Including Micellization with Gemini Surfactants. Indian J of Pharmaceutical Education and Research. 2018;52(3):334-41. 